\title{
InNovative Hill Start Assistance Device
}

\author{
Paulo Burger, Claudinei Buzinaro, Alberto Bucci \\ Magneti Marelli Powertrain \\ E-mails: paulo.burger@magnetimarelli.com, claudinei.buzinaro@magnetimarelli.com, \\ alberto.bucci@magnetimarelli.com
}

\begin{abstract}
The hill start in manual or automated manual transmission vehicles is faced by most users as inconvenient situation. In the time interval between brake pedal releasing and gas pedal pressing, the car might roll backwards, causing an unsafe or at least uncomfortable situation. The device currently presented offers a simple solution that assists the driver in such situation, keeping the vehicle stopped while the driver releases the brake pedal and requests enough engine torque to safely start in hill condition. The simplicity of the device allows its assembly in manual, automated manual or even automatic transmission cars, with or without anti-lock brake system and it also ensures its use from the lowest to the top range of vehicles. The operation principle is based on a directional valve assembled in the brake line which is only activated when all the logic conditions are redundantly checked on a hill start situation. An accelerometer is used to identify the exact hill inclination in which is the vehicle and calculate the required engine torque for a safe start.
\end{abstract}

\section{INTRODUCTION}

A complex arrangement of factors defines whether or not a new vehicle will become a market success. The continuously more restrictive emission regulation and the increasing concernment regarding fuel consumption have been pushing the powertrain development for new standards on every vehicle launch. Aiming efficiency, several innovative technologies have reached production lines such as downsized engines, hybrid cars and large variety of gearboxes with different principles and several gear ratios.

Safety technologies have equally been largely spread over the past few years. In Brazil, the use of anti-lock brake system is defined as obligatory for all vehicles manufactured after January 1st 2014 [1]. The same definition applies to the use of airbags [2].

Despite the legislation, which defines the minimum requirements, the automotive market itself is even more demanding for safety matters. To ensure competitiveness, the basic rule for vehicles is to offer the larger number of features for the lowest price, ensuring the value from the costumers' perspective. A frequent situation that causes concern for drivers is starting the car in a hill situation without any rollback. This action requires considerable expertise and concentration from the driver and may cause stress in critical hill situations such as maneuvering is tight parking spaces or heavy traffic with frequent stops and starts. If the car rolls to the unintended direction, there is the risk of accident. The vehicle parts are also 
exposed to abnormal wear if the driver creates excessive clutch slipping during the start. The situations listed above are more applicable to cars with manual or automated manual transmission gearboxes. Nevertheless, automatic transmission vehicles may also be submitted to rollback on hill start if the residual transmission torque is not high enough to keep the vehicle completely stopped.

In order to provide assistance to the driver in hill start situation, the device herein presented was developed to prevent the vehicle from rolling in the time interval between brake pedal release and sufficient gas pedal pressing. The purpose of the development is to offer a simple - and therefore massively applicable - solution a very frequent issue faced on a frequent basis by drivers.

\section{OVERALL CONCEPT}

The system consists of a hydraulic device managed by an electronic system that keeps the vehicle brake activated for a given time to assist the driver in hill starting condition. In such condition, the vehicle remains stopped in the time interval between brake pedal release and gas pedal pressing, preventing movement to the unintended direction.

The simplicity of the device is based on the principle that the braking force is generated by the driver himself, when stopping the vehicle. The device only holds the braking pressure and no electric pump is therefore required, significantly reducing the complexity, failure sources and costs.

A hydraulic unit interfaces with specific electronic components, managed by software in the Control Unit. Several layouts are possible for the Control Unit, which varies according to the architecture of each vehicle. Depending on the application, the Engine Control Unit (ECU) or the Transmission Control Unit (TCU) might possibly assume the role of managing the complete system; therefore no additional physical Control Unit is necessary. This integration depends on the existing unit's software/hardware capability and compatibility with the additional system.

\subsection{Hydraulic logics}

One of the possible hydraulic arrangements is show in Figure 1. A single body hydraulic group is assembled in the brake lines, between the master-cylinder and the antilock brake system's (ABS) unit. This layout was chosen because it illustrates a predominant choice in automobiles brake systems. The main components are the brake booster coupled to the master-cylinder from which derive two independent brake lines. Each line is connected to the device and remains independent to its output, which is then connected to the ABS module. From the ABS module, four independent brake lines go for each wheel brake system. As previously mentioned, the presence of ABS system is not mandatory for the device operation.

Within the hydraulic group, the hydraulic concept is schematically represented by two On/Off solenoid valves and two directional valves. This representation ensures the operational logics, although is does not necessarily correspond to the actual physical layout of the device. For example, a single solenoid valve may integrate the On/Off and the directional functions in the same body. Additionally, the On/Off solenoid 
valve might be replaced by a proportional valve to smoothen the activation and deactivation.

The operational logic is divided in four different phases. Initially, when the device is inactive, the brake system works normally without any intervention from the device. The solenoid valves are inactive, therefore the brake lines suffer no intervention, as shown in Figure 1.

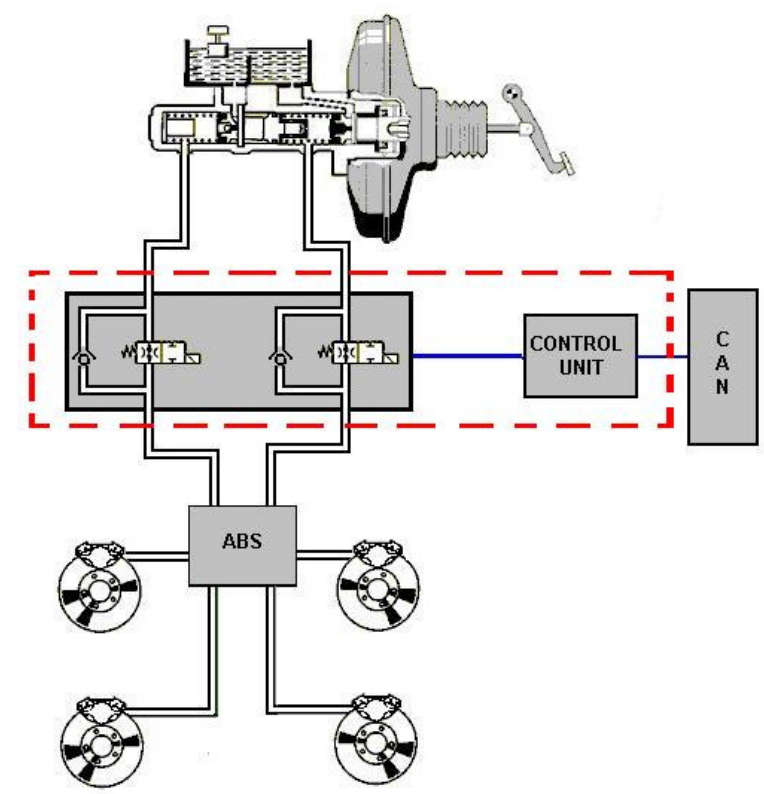

Figure 1. Phase 1: Brake system inactive and device inactive

When the driver presses the brake pedal, the brake oil pressure is fully transmitted throughout the brake lines, as shown in Figure 2. If the vehicle is completely stopped, the system logics checks all requirements needed to activate the solenoid valves. The requirements are detailed in the "Software logics". If all checks have positive result, the solenoid valves are then activated. 


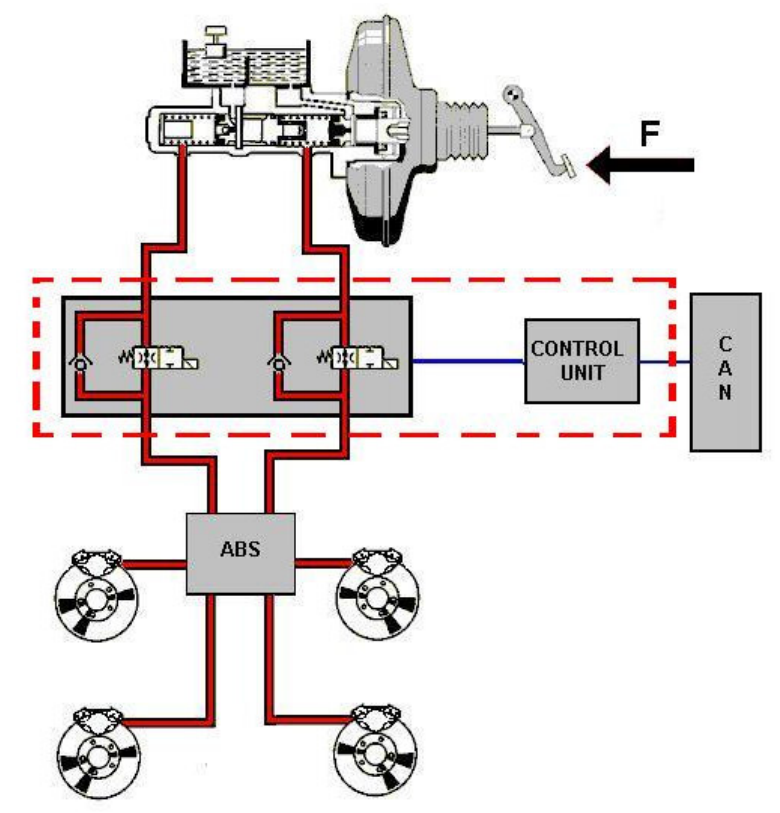

Figure 2. Phase 2: Brake force applied by the driver to stop the vehicle on hill condition; system logics check requirements for activation

When the solenoid valves are activated, as in Figure 3, the oil flow trough the hydraulic device assumes a directional logic i.e., the oil can flow from the mastercylinder to ABS but not otherwise. In this condition, the maximum brake pressure applied by the driver remains in the wheel brake system.

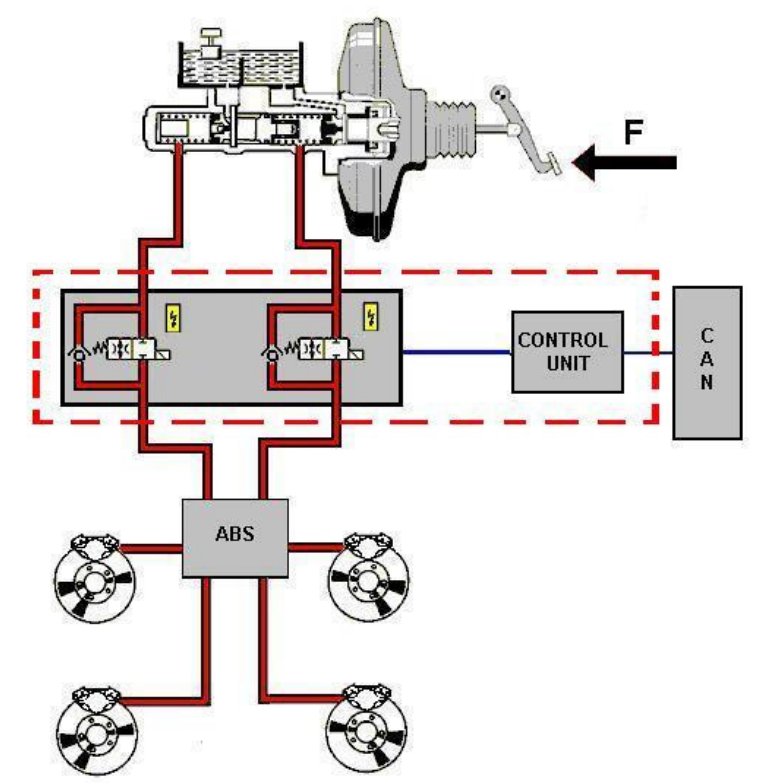

Figure 3. Phase 3: The system logics detect that the vehicle conditions meet all required hill condition and activates the solenoid valves 
Finally, when the driver releases the brake pedal, the vehicle remains stopped because the solenoid valves are activated and the oil pressure remains between the device and the brake of each wheel, as shown in Figure 4, according to the software logics. In this phase, the driver has enough time to press the gas pedal, modulate the clutch (in manual transmission cars) and safely start the vehicle without rollback.

When the solenoid valves are deactivated, the brake system goes to Phase 1, represented in Figure 1, as in a conventional braking system. The deactivation logics are explained in the "Software logics" section.

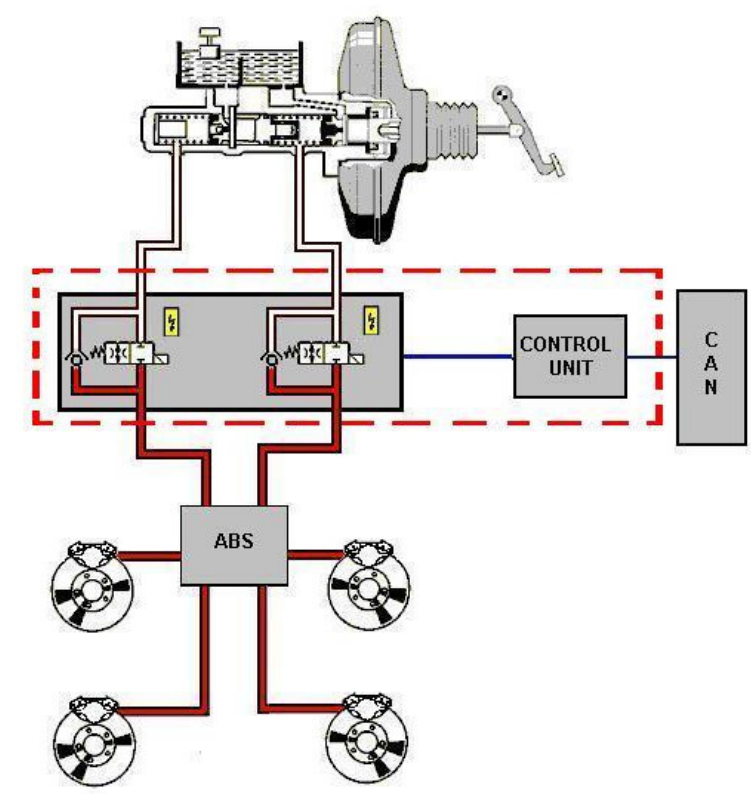

Figure 4. Phase4: The brake pedal is released by the user and the brake force remains applied to the wheels

\subsection{Software logics}

A diversified list of software strategies can be used to ensure the correct functionality and the proper safety requirements for each application. Nevertheless, the basic function of the Start Assistance Device is ensured by signals commonly available in most production vehicles. The source or availability of each signal is not part of the present scope of work because it varies for each application. The basic software logics are described in the present work, which does not eliminate the possibility to add more complex or redundant strategies to the system or even change part of the logics according to specific requirements.

\subsubsection{System activation}

The basic checks for the system activation are: vehicle completely stopped for a minimum threshold of time, road slope above a threshold, engine on and brake pressed. All thresholds are set by calibration for each application. If the engaged gear signal is available, the system only activates in downhill if 
Reverse gear is engaged; likewise, if the vehicle is in uphill condition, the system only activates if the Reverse gear is not engaged.

After these requirements for system activation are confirmed, the solenoid valves are then activated, going to Phase 3, represented in Figure 3.

\subsubsection{System active}

When the vehicle is stopped in a slope, the software calculates the required engine torque needed to keep the vehicle stand still, compensating the gravity force component in the direction of the slope.

\subsubsection{System deactivation}

When the driver releases the brake pedal, the system goes to Phase 4 (Figure 4), remaining active for an adjustable time and keeping the vehicle stopped on the hill, waiting for the user's starting action. As soon as the driver requests enough engine torque to safely start on the hill, the system immediately deactivates the solenoid valves and releases the brake pressure, allowing the vehicle motion in the intended direction.

If no action if performed by the driver when the system is on Phase 4 (Figure 4), the system is deactivated after a timeout, allowing vehicle rollback afterwards. On the other hand, if the driver begins to slightly press the gas pedal, the software extends the deactivation timeout to allow the driver to continue pressing the gas pedal until the engine delivers the needed torque. If the engine does not reach the required torque to start on the hill, the system is then deactivated after a second adjustable timeout.

In order to meet safety requirements, the system can have specific strategies to become inactive, always alerting the driver in case this happens. For example, if any vital input for the system presents a faulty behavior, such as vehicle speed or slope signal, the system must become inactive for lack or reliable information. The safety-related software strategies are not part of the present scope and will be detailed in a specific work.

\section{CONCLUSIONS}

The present work describes a system which has a simple concept of operation but offers an extremely useful benefit for the driver. Assisting the user in hill start situation simultaneously enhances vehicle safety and comfort. The simplicity of the presented concept enforces its immediate commercial potential, allowing its application from the cheapest models to top-end of a car manufacturer portfolio. These aspects converge with the fast evolution in the automotive technologies required by a highly demanding market. 


\section{REFERENCES}

1. SILVA A. P. et al. Resolução n³12, de 03 de Abril de 2009 - DENATRAN. Available at http://www.denatran.gov.br/download/Resolucoes/RESOLUCAO_CONTRAN_312_09.pdf . Access on May $10^{\text {th }} 2013$.

2. SILVA A. P. et al. Resolução n ${ }^{\circ} 311$, de 03 de Abril de 2009 - DENATRAN. Available at http://www.denatran.gov.br/download/Resolucoes/RESOLUCAO_CONTRAN_311_09.pdf. Access on May $10^{\text {th }} 2013$. 